\title{
Biodegradable and thermosensitive micelles inhibit ischemia-induced postoperative peritoneal adhesion
}

This article was published in the following Dove Press journal:

International Journal of Nanomedicine

5 February 2014

Number of times this article has been viewed

\section{Qinjie Wu \\ Ling Li \\ Ning Wang \\ Xiang Gao \\ Bilan Wang \\ Xinyu Liu \\ Zhiyong Qian \\ Yuquan Wei \\ Changyang Gong}

State Key Laboratory of Biotherapy and Cancer Center, West China Hospital, West China Medical School, Sichuan University, Chengdu, People's Republic of China
Correspondence: Changyang Gong State Key Laboratory of Biotherapy and Cancer Center, West China Hospital, Sichuan University, Chengdu 61004I, People's Republic of China

Tel +862885I64063

Fax +862885164060

Email chygongl4@I63.com
Abstract: Ischemia-induced adhesion is very common after surgery, and leads to severe abdominal adhesions. Unfortunately, many existing barrier agents used for adhesion prevention have only limited success. The objective of this study is to evaluate the efficacy of biodegradable and thermosensitive poly( $\varepsilon$-caprolactone)-poly(ethylene glycol)-poly( $\varepsilon$-caprolactone) (PCL-PEG-PCL) micelles for the prevention of postoperative ischemia-induced adhesion. We found that the synthesized PCL-PEG-PCL copolymer could self-assemble in an aqueous solution to form micelles with a mean size of $40.1 \pm 2.7 \mathrm{~nm}$ at $10^{\circ} \mathrm{C}$, and the self-assembled micelles could instantly turn into a nonflowing gel at body temperature. In vitro cytotoxicity tests suggested that the copolymer showed little toxicity on NIH-3T3 cells even at amounts up to $1,000 \mu \mathrm{g} / \mathrm{mL}$. In the in vivo test, the postsurgical ischemic-induced peritoneal adhesion model was established and then treated with the biodegradable and thermosensitive micelles. In the control group $(\mathrm{n}=12)$, all animals developed adhesions (mean score, $3.58 \pm 0.51$ ), whereas three rats in the micelles-treated group $(\mathrm{n}=12)$ did not develop any adhesions (mean score, $0.67 \pm 0.78$; $P<0.001$, Mann-Whitney $U$-test). Both hematoxylin and eosin and Masson trichrome staining of the ischemic tissues indicated that the micelles demonstrated excellent therapeutic effects on ischemia-induced adhesion. On Day 7 after micelle treatment, a layer of neo-mesothelial cells emerged on the injured tissues, which confirmed the antiadhesion effect of the micelles. The thermosensitive micelles had no significant side effects in the in vivo experiments. These results suggested that biodegradable and thermosensitive PCL-PEG-PCL micelles could serve as a potential barrier agent to reduce the severity of and even prevent the formation of ischemia-induced adhesions.

Keywords: postsurgical adhesion, poly( $\varepsilon$-caprolactone)-poly(ethylene glycol)poly( $\varepsilon$-caprolactone) (PCL-PEG-PCL), surgical complications, barrier agent

\section{Introduction}

Peritoneal adhesion, a result of the wound-healing process, is a common complication after abdominal or pelvic surgery. Postoperative adhesion can lead to a variety of adverse consequences, such as chronic debilitating pain, female infertility, small bowel obstruction, and difficulty with future operations, ${ }^{1,2}$ which not only cause pain but also create a great medical and economic burden to patients. Therefore, preventing or minimizing adhesion formation is the goal of many researchers.

There are many factors involved in the formation of postoperative adhesions, including trauma, ischemia, foreign bodies, and infections. ${ }^{1}$ Sutures and ligation are unavoidable in almost all abdominal and pelvic surgeries; such operations will induce local tissue ischemia, which can cause inflammatory responses of the peritoneum, thus leading to 
the formation of adhesions. ${ }^{3}$ The formed adhesions can attach to various structures in the peritoneum, such as the omentum, small intestine, large intestine, and even the liver, which creates an extremely hazardous situation for the patient. ${ }^{4}$ Thus, there is an urgent need to prevent or reduce ischemia-induced postoperative peritoneal adhesion formation.

So far, many approaches have been advocated to minimize or prevent the development of postoperative adhesions, including anti-inflammatory agents, antibiotics, fibrinolytic agents, and chemical and physical barriers, ${ }^{1}$ which can be classified as pharmaceutical agents and barrier agents. Owing to rapid intraperitoneal clearance, many antiadhesion drugs are difficult to incorporate into clinical practice. ${ }^{5}$ Another way to protect against adhesion formation is through the use of barrier agents, including viscous polymer solution, synthetic solid membrane, and in situ cross-linked hydrogel, which can separate two injured regions during peritoneal healing. Unfortunately, none of these measures have proven to be uniformly effective under all surgical conditions. For the application of polymer solution, like carboxymethylcellulose and sodium hyaluronate, their residence time at the administration site is relatively brief, which may greatly compromise their therapeutic effects as barrier systems. ${ }^{6}$ For the synthetic solid membrane, such as Interceed, Seprafilm, and PTFE, their residence time is long (over 30 days), but this much longer residence time is also one of the possible negative aspects. In addition, the application of these synthetic solid barriers is not convenient to handle or fix the injured tissues, particularly in laparoscopic surgery. ${ }^{7}$ Recently, the crosslinked hydrogel system has overcome some of the limitations mentioned above, but it needs a relatively long gelation time (5 to 45 minutes), and is considered to be cumbersome. ${ }^{8}$

In our previous work, we successfully synthesized poly( $\varepsilon$-caprolactone)-poly(ethylene glycol)-poly( $\varepsilon$-caprolactone) (PCL-PEG-PCL) triblock copolymers in one step and prepared injectable and thermosensitive micelles, which proved to be biodegradable and biocompatible. ${ }^{9,10} \mathrm{We}$ found that the obtained PCL-PEG-PCL micelles worked well as a novel antiadhesion barrier for preventing formation of sidewall defect-cecum abrasion-induced abdominal adhesion. ${ }^{11}$ Compared to sidewall defect-cecum abrasioninduced adhesion, ischemia-induced adhesion after surgery is more common and will lead more severe abdominal adhesions. However, whether thermosensitive PCL-PEGPCL micelles have an ability to prevent formation or reduce severity of ischemia-induced adhesion is still unclear. In this study, the efficacy of thermosensitive PCL-PEGPCL micelles with regard to antiadhesion formation was assessed in a rat ischemia-induced adhesion model, which induced more severe adhesions and better mimicked clinical circumstances.

\section{Materials and methods Materials, cells, and animals}

$\varepsilon$-Caprolactone ( $\varepsilon$-CL), poly(ethylene glycol) (PEG, $\left.M_{\mathrm{n}}=1,000\right)$, stannous octoate $\left(\mathrm{Sn}(\mathrm{Oct})_{2}\right)$, and 3-(4,5dimethylthiazol-2-yl)-2,5-diphenyl tetrazolium bromide (MTT) were purchased from Sigma-Aldrich (St Louis, MO, USA). RPMI-1640 medium and fetal bovine serum was obtained from Life Technologies (Carlsbad, CA, USA). All regents were used without further purification, and the materials were analytical reagent grade and used as received.

Murine NIH-3T3 fibroblast cell line was purchased from the American Type Culture Collection (Manassas, VA, USA) and cultured in RPMI-1640 medium with 10\% fetal bovine serum.

Female Sprague Dawley rats, weighing 220 \pm 20 g, were purchased from the Laboratory Animal Center of Sichuan University and quarantined for 1 week. All animal care and experimental procedures were carried out according to Principles of Laboratory Animal Care.

\section{Synthesis and purification of PCL-PEG-PCL triblock copolymer}

PCL-PEG-PCL triblock copolymer was synthesized and purified as described in our previous work. ${ }^{9}$ Briefly, calculated amounts of $\varepsilon-\mathrm{CL}, \mathrm{PEG}$ and $\mathrm{Sn}(\mathrm{Oct})_{2}$ were introduced into a dry glass ampoule under a nitrogen atmosphere; $\mathrm{Sn}(\mathrm{Oct})_{2}$ was used as a catalyst. The reaction system was kept at $130^{\circ} \mathrm{C}$ for 6 hours, degassed under vacuum for another hour, and the resultant copolymer was cooled to room temperature. The obtained PCL-PEG-PCL triblock copolymers were dissolved in dichloromethane and precipitated with petroleum. After being filtered and vacuum dried to constant weight at room temperature, the purified polymers were kept in desiccators for further use. The obtained copolymer was characterized by ${ }^{1} \mathrm{H}$ nuclear magnetic resonance spectroscopy $\left({ }^{1} \mathrm{H}-\mathrm{NMR}\right.$, Varian 400 spectrometer, Varian, Palo Alto, CA, USA), Fourier transform infrared spectroscopy (Nicolet $200 \mathrm{SXV}$, Thermo Fisher Scientific, Waltham, MA, USA), and gel permeation chromatography (Agilent 110 HPLC, Agilent Technologies, Santa Clara, CA, USA).

\section{Preparation and characterization of PCL-PEG-PCL micelles}

The preparation of PCL-PEG-PCL micelles was carried out as described previously. ${ }^{12,13}$ In brief, the purified 
PCL-PEG-PCL triblock copolymers were dissolved in deionized water at $55^{\circ} \mathrm{C}$ at the concentration of $20 \mathrm{wt} \%$ and cooled to $4^{\circ} \mathrm{C}$ to form micelles.

Particle size and zeta potential of the micelles in aqueous solution were detected at $10^{\circ} \mathrm{C}$ with a laser diffraction particle size analyzer (Nano-ZS 90, Malvern Instruments, Malvern, UK). In addition, the sol-gel-sol phase transition behavior of the micelles was investigated by the tube-inverting method. ${ }^{14}$ Briefly, a sample was loaded into a $4 \mathrm{~mL}$ tightly screw-capped vial and then heated; the heating rate was $1^{\circ} \mathrm{C} /$ minute. The sol-gel-sol phase transition was visually observed by inverting the vials.

\section{Cytotoxicity of the micelles in vitro}

To assess the cytotoxicity of obtained micelles, MTT assay was carried out, and NIH-3T3 cells were used. In detail, NIH3 T3 cells were plated in 96-well plates $\left(1 \times 10^{4}\right.$ each well $)$ and incubated for 24 hours. Then, according to calculated concentration (from 0 to $1,000 \mu \mathrm{g} / \mathrm{mL}$ ), the PCL-PEG-PCL micelles were serially diluted and added into each well, followed by incubation for 24 hours or 48 hours; MTT was then added $(5 \mathrm{mg} / \mathrm{mL})$. After the precipitated formazan formed, $200 \mu \mathrm{L}$ of dimethyl sulfoxide was added into each well and the absorbance at $570 \mathrm{~nm}$ was measured by an enzymelinked immunosorbent assay reader (Multiskan Mk3, Thermo Fisher Scientific). The mean percentage of cell survival relative to that of untreated cells was estimated from data of six individual experiments. All data were expressed as the mean \pm standard deviation.

\section{Antiadhesion assessment on ischemic-induced peritoneal adhesion model in vivo}

To evaluate the effects of thermosensitive micelles on peritoneal adhesion formation, the ischemic-induced peritoneal adhesion model was established in adult Sprague Dawley rats. ${ }^{15}$ In brief, the rats were anesthetized with ketamine hydrochloride (60 mg/kg body weight) and then placed in a supine position. After the abdominal surface was shaved with a surgical blade, all rats underwent a median laparotomy incision with a total length of $4 \mathrm{~cm}$. Then, four ischemic buttons spaced $1 \mathrm{~cm}$ apart were created on the right side lateral sidewall of the parietal peritoneum in each rat by grasping $5 \mathrm{~mm}$ of peritoneum with a hemostat and ligating the base of the segment with $4 / 0$ silk suture. Then rats were randomly assigned to the normal saline (NS)-treated group (control group, $\mathrm{n}=12$ ) or the PCL-PEG-PCL micelles treatment group $(n=12)$. After surgery, the ischemic buttons were treated with
NS or micelles ( $1 \mathrm{~mL}, 20 \% \mathrm{wt})$ respectively, and the incision was closed in two layers with 3/0 surgical silk suture. All animals were housed in filter-topped cages (one rat per cage) with free access to animal chow and water.

After 7 days, all rats were sacrificed and the severity of adhesions in each animal was evaluated according to the standard adhesion scoring system, ${ }^{4,16}$ which was based on the number of ischemic buttons with attached adhesions as follows: score 0 , no adhesion; score 1 , only one thin filmy adhesion; score 2, two firm adhesions; score 3, three dense adhesions; score 4, four or more dense adhesions. The adhesions were quantified in a blinded fashion.

\section{Histopathological assessment}

On Day 7 after surgery, the specimens were taken carefully from the ischemic buttons and adhesion tissues. The obtained tissues were fixed by $4 \%$ paraformaldehyde in phosphate buffered saline (PBS) for 48 hours, embedded in paraffin, and sectioned. Samples were then stained with hematoxylin and eosin (H\&E) for general histopathological examination and Masson trichrome staining for collagen deposition evaluation. All tissue slides were analyzed by light microscopy (Olympus BX 45, Olympus Corporation, Tokyo, Japan) by two pathologists in a blinded manner.

\section{Scanning electron microscope analysis}

For microscopic analysis, the obtained tissues were fixed with prechilled $2.5 \%$ glutaraldehyde in $0.1 \mathrm{MPBS}$ and dehydrated in a critical point apparatus. After a gold sputter coating, they were examined by scanning electron microscope (JSM5900LV, JEOL, Tokyo, Japan).

\section{Toxicity assessment}

To evaluate possible side effects of PCL-PEG-PCL micelles on the animals, all rats were observed after surgery. The observation included assessment of their general conditions (activity, energy, hair, feces, behavioral pattern, and other clinical signs), body weight, mortality, food intake, and water intake. Seven days after treatment, major organs, including heart, liver, spleen, lung, and kidney, were harvested and fixed with 4\% paraformaldehyde in PBS for 48 hours, embedded in paraffin, then stained with $\mathrm{H} \& \mathrm{E}$ for histopathological analysis.

\section{Statistical analysis}

Data were analyzed using SPSS 15.0 software (IBM Corporation, Armonk, NY, USA). Adhesion scores did not follow a normal distribution; therefore, statistical inferences were 
made using Mann-Whitney $U$-tests or the Fisher's exact test. $P<0.05$ on a two-tailed test was considered statistically significant.

\section{Results and discussion}

\section{Characterization of PCL-PEG-PCL micelles}

PCL-PEG-PCL triblock copolymers were prepared by ringopening copolymerization of $\varepsilon$-CL initiated by PEG using $\mathrm{Sn}(\mathrm{Oct})_{2}$ as catalyst. Molecular weight of PCL-PEG-PCL triblock copolymer was 3,100 , which was determined by ${ }^{1}$ H-NMR.

The copolymer was self-assembled into micelles in aqueous solution, and a laser diffraction particle size analyzer (Nano-ZS 90, Malvern Instruments) was used to detect the particle size and zeta potential of the prepared PCL-PEGPCL micelles at $10^{\circ} \mathrm{C}$. As shown in Figure 1, the mean particle size and zeta potential of the micelles were $40.1 \pm 2.7 \mathrm{~nm}$ (Figure 1A) and $-0.57 \pm 0.49 \mathrm{mV}$ (Figure 1B), respectively.

\section{Phase transition \\ of thermosensitive micelles}

The PCL-PEG-PCL micelles exhibited a special sol-gel phase transition behavior, shown in Figure 2A. When the PCL-PEG-PCL concentration was above the critical gelation concentration, the micelles were an injectable free-flowing solution at ambient temperature, but converted instantly into a nonflowing gel at around physiological temperature. The mechanism of the sol-gel phase transition may associate with micelle growth and aggregating. As shown in Figure 2B, particle size of the micelle solution was determined in different temperatures. When the temperature increased from $10^{\circ} \mathrm{C}$ to $20^{\circ} \mathrm{C}$, the particle size of micelles increased slightly. However, as the temperature increased form $20^{\circ} \mathrm{C}$ to $40^{\circ} \mathrm{C}$, the particle size of micelles dramatically increased.

\section{Cytotoxicity of the micelles in vitro}

Cytotoxicity of the micelles was evaluated by cell viability assay using NIH-3T3 cells for 24 hours and 48 hours. As shown in Figure 3, with the increase of micelle concentration, viability of NIH-3T3 cells decreased accordingly at the same incubation time. With the same micelle concentration, the viability of NIH-3T3 cells decreased with incubation time increase. The cell viability was higher than $77 \%$, even when the input micelle concentration was up to $1,000 \mu \mathrm{g} / \mathrm{mL}$ and incubation lasted for 48 hours. A cytotoxicity study indicated that the micelles prepared in this work were biocompatible with low cytotoxicity, which suggested that the thermosensitive micelles could be a safe antiadhesion barrier agent.
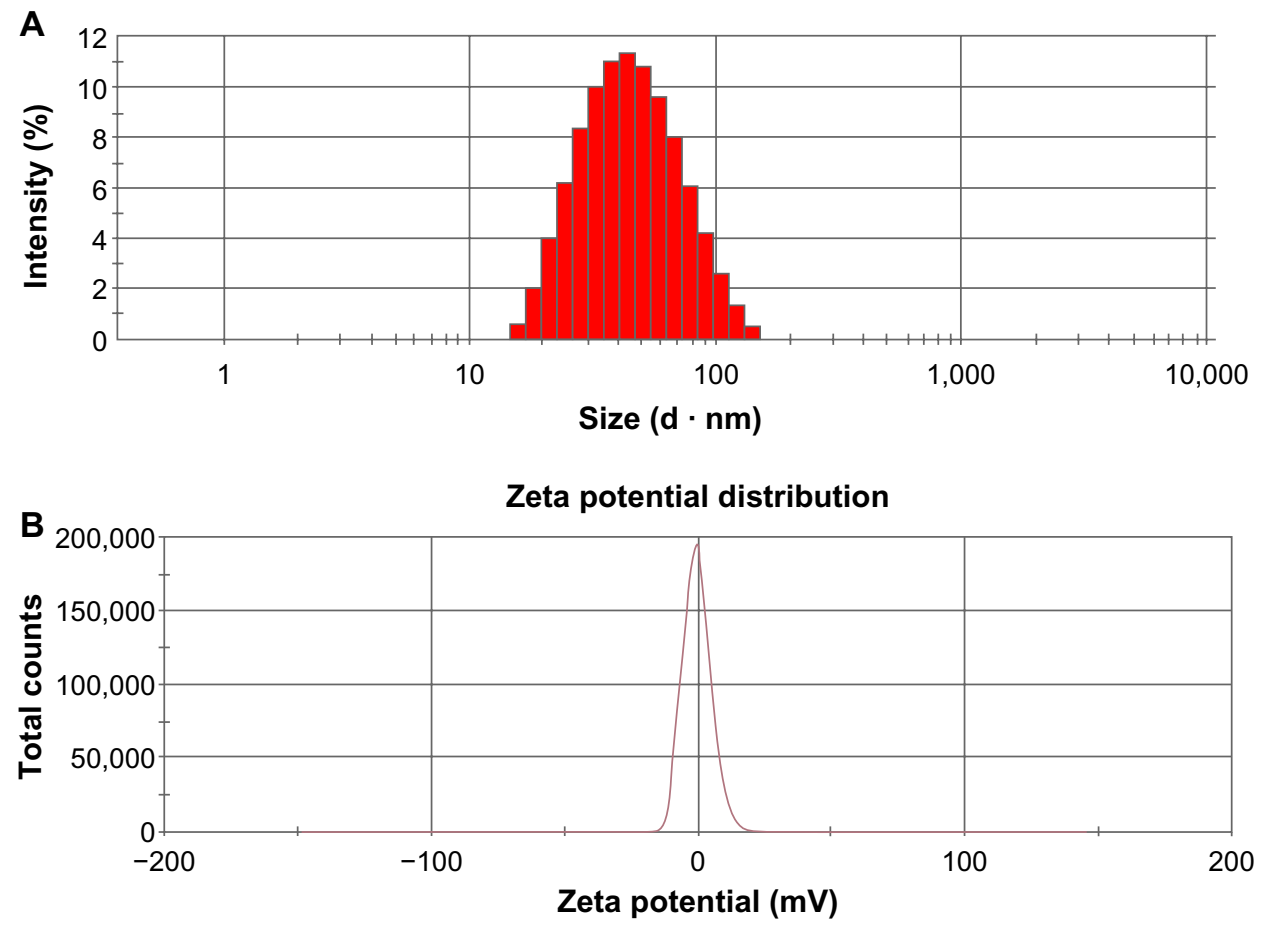

Figure I Characterization of PCL-PEG-PCL micelles.

Notes: (A) Particle size of micelles at $10^{\circ} \mathrm{C}$. (B) Zeta potential of micelles at $10^{\circ} \mathrm{C}$.

Abbreviation: PCL-PEG-PCL, poly( $\varepsilon$-caprolactone)-poly(ethylene glycol)-poly( $\varepsilon$-caprolactone). 

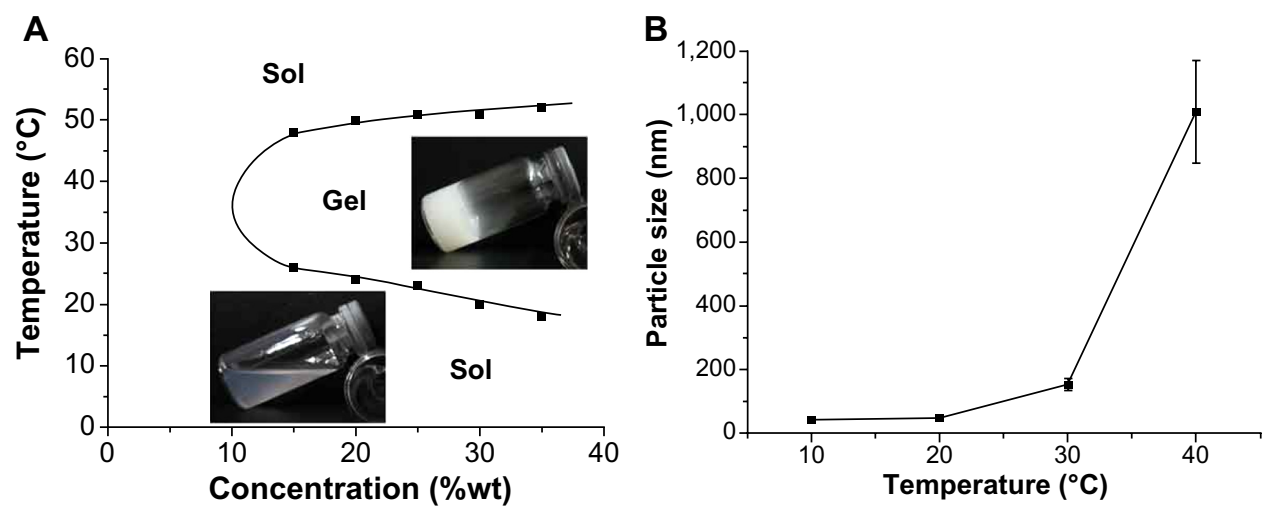

Figure 2 Thermosensitivity of prepared PCL-PEG-PCL micelles.

Notes: (A) Sol-gel phase transition of the thermosensitive micelles as a function of temperature. (B) Particle size of micelles at different temperatures. Abbreviation: PCL-PEG-PCL, poly( $\varepsilon$-caprolactone)-poly(ethylene glycol)-poly( $\varepsilon$-caprolactone).

\section{Antiadhesion assessment of the thermosensitive micelles in vivo}

To evaluate the antiadhesion effect of the PCL-PEG-PCL micelles, the ischemic-induced postoperative peritoneal adhesion model in SD rats was employed. As shown in Figure 4, four ischemic buttons spaced $1 \mathrm{~cm}$ apart were created successfully by surgery on the right side lateral sidewall of each rat and then treated with micelles or NS. On postoperative Day 7, all rats were sacrificed and the extent of adhesion in each group was assessed (Table 1 and Figure 5). For adhesions score and percentage, there was a significant difference between the control group and the micelles-treated group (mean score, $3.58 \pm 0.51$ in NS group vs $0.67 \pm 0.78$ in micelles-treated group; $P<0.001$, Mann-Whitney $U$-test). In the control group, $100 \%$ of rats formed very severe adhesions (score 4 or 3). Figure 5A shows that one adhesion attached to omentum and scored 4 in the NS group. In contrast, only $50 \%$ of the rats in the micelles group formed adhesions,

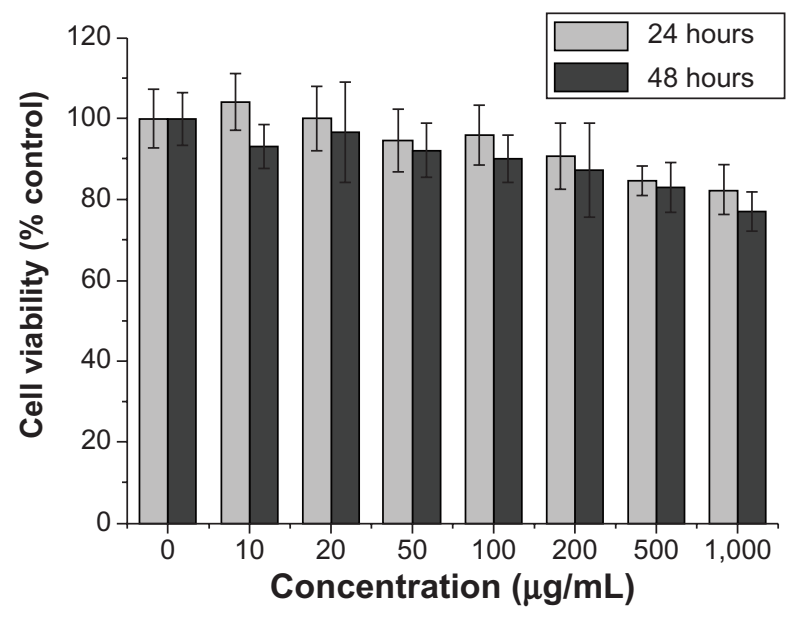

Figure 3 Cytotoxicity of the micelles in vitro using MTT assay. Abbreviation: MTT, 3-(4,5-dimethylthiazol-2-yl)-2,5-diphenyl tetrazolium bromide. which were less severe (score 1 or 2) than those of the control group. Figure 5B shows that no adhesion was observed after micelles treatment. Although a few adhesions formed in the micelles group, the adhesions were less dense and filmier than those in the control group. These results indicated that the thermosensitive micelles could reduce the severity and even prevent formation of ischemia-induced adhesion.

\section{Histopathological assessment}

Tissue samples from ischemic buttons in each group were collected and stained with $\mathrm{H} \& \mathrm{E}$ and Masson trichrome staining. As shown in Figure 6A and 6B, in the control group, ischemic buttons were formed with silk sutures buried in the fibrous tissues. Connective tissues of the omentum were adhered to the surface and peripheral area of the ischemic button. Dense adhesions were observed between the ischemic button and connective tissues, and no mesothelial cells were observed on the surface of the ischemic button.

$\mathrm{H} \& \mathrm{E}$ and Masson trichrome staining of ischemic buttons in the micelles-treated group are shown in Figure 6C-6F. A similar ischemic button was also formed in the micellestreated group, but no adhesions were observed. In the

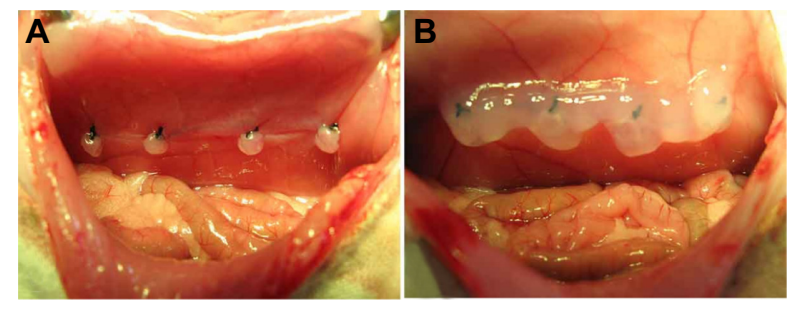

Figure 4 Establishment and treatment of ischemic-induced postoperative peritoneal adhesion model in Sprague Dawley rats.

Notes: (A) Normal saline-treated group as control group. (B) PCL-PEG-PCL micelles-treated group.

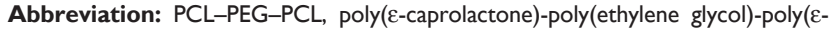
caprolactone). 
Table I Evaluation of peritoneal adhesions in the mouse model

\begin{tabular}{lll}
\hline Adhesion score & Control $(\mathbf{n}=\mathbf{|} \mathbf{2})$ & Micelles $(\mathbf{n}=\mathbf{|} \mathbf{2})$ \\
\hline Score 4 & $7(58.3 \%)$ & 0 \\
Score 3 & $5(41.7 \%)$ & 0 \\
Score 2 & 0 & $2(16.7 \%)$ \\
Score I & 0 & $4(33.3 \%)$ \\
Score 0 & 0 & $6(50 \%)$ \\
Mean score & $3.58 \pm 0.51$ & $0.67 \pm 0.78^{*}$ \\
\hline
\end{tabular}

Note: In comparison with the control group, treatment with micelles significantly prevented adhesion formation $(* P<0.00 I)$.

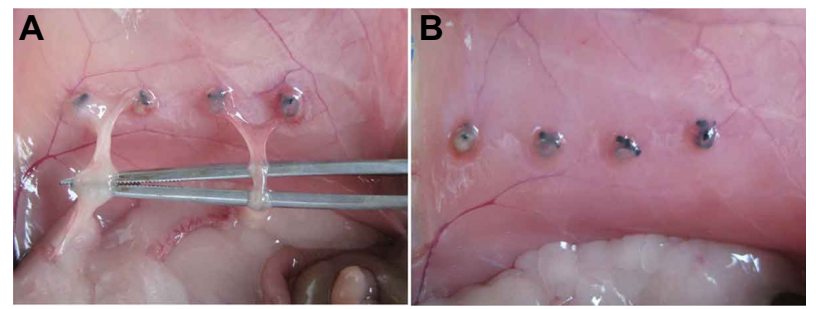

Figure 5 Evaluation of ischemia-induced adhesion prevention on postoperative Day 7. Notes: (A) Adhesions were observed in control group, which scored 4. (B) No adhesion was observed in micelles-treated group, which scored 0 .

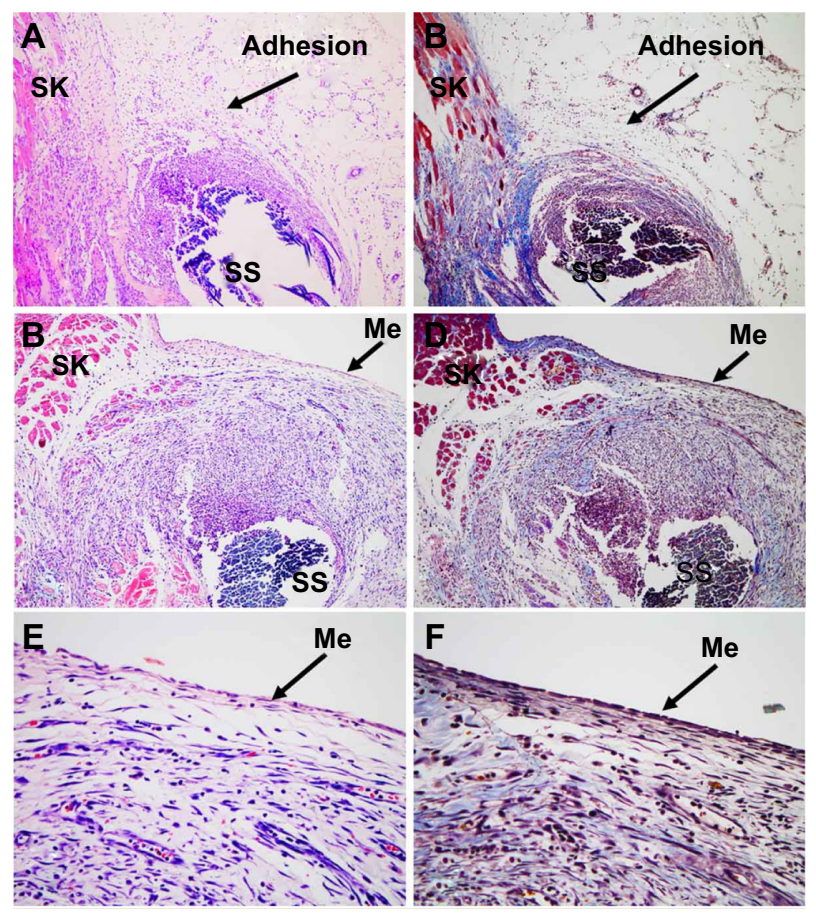

Figure 6 Histopathological analyses of tissues from control group and micellestreated group.

Notes: (A) Adhesion tissues from control group stained with H\&E (I00X). (B) Adhesion tissues from control group stained with Masson (100x). (C) Tissues from micelles-treated group stained with H\&E (I00X). (D) Tissues from micellestreated group stained with Masson (I00X). (E) The appearance of neo-mesothelial cells in tissues stained with H\&E on Day 7 after micelles treatment $(400 \times)$. (F) The appearance of neo-mesothelial cells in tissues stained with Masson on Day 7 after micelles treatment $(400 \times)$. Black arrows in each slide showed the position of adhesion or mesothelial cells in each figure.

Abbreviations: H\&E, hematoxylin and eosin; Me, mesothelial cells; SK, abdominal wall skeletal muscle; SS, silk suture. enlarged imaging (Figure 6E and 6F), a monolayer of neo-mesothelial cells was observed, which indicated that the formed ischemic button recovered without concern of adhesion formation in the future.

\section{Remesothelization of micelles- treated tissues}

The peritoneum is composed of a monolayer of mesothelial cells with distinctive microvilli, which were elongated, flattened, and looked like squamous cells under SEM. As shown in Figure 7, we observed the restoration of the mesothelial cell layer of the ischemic button by SEM. On Day 7 after micelles treatment, many elongated, flattened, squamous-like neo-mesothelial cells were covered on the surfaces of the ischemic button, and a lot of microvilli were observed on the surface of neo-mesothelial cells. Due to mesothelial cells were the most important components of the peritoneum, ${ }^{17-19}$ the appearance of neo-mesothelial cells had the benefit of repairing the injured peritoneal wall, which prevents peritoneal adhesion. These results indicated that mesothelial cells, which were on the surface of the ischemic button, have restored very well, and PCL-PEG-PCL micelles could prevent ischemia-induced adhesion effectively.

\section{Systemic toxicity analysis}

To evaluate the potential toxicity of PCL-PEG-PCL micelles, general conditions, body weight, and food and water intake were monitored. During our observation period, no death occurred, and no toxic response was found in rats. The animals displayed normal behavior, free movement, and shining hair. In addition, there was no significant difference in body weight (Figure 8A), food intake (Figure 8B) and water intake (Figure 8C) between rats in the micelles group and rats in the NS group $(P>0.05, P>0.05$, and $P>0.05$, respectively).

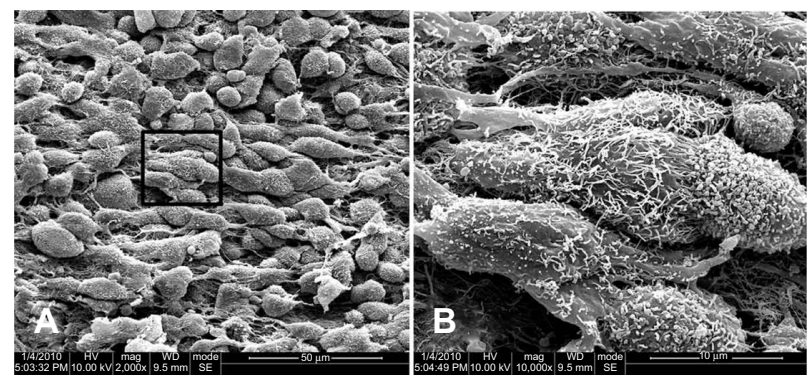

Figure 7 SEM images of neo-mesothelial cells on Day 7 after micelle treatment. Notes: Images are taken at 2,000x (A), and 10,000x magnification (B). Inset shown in $\mathbf{A}$ is magnified in $\mathbf{B}$.

Abbreviation: SEM, scanning electron microscope. 

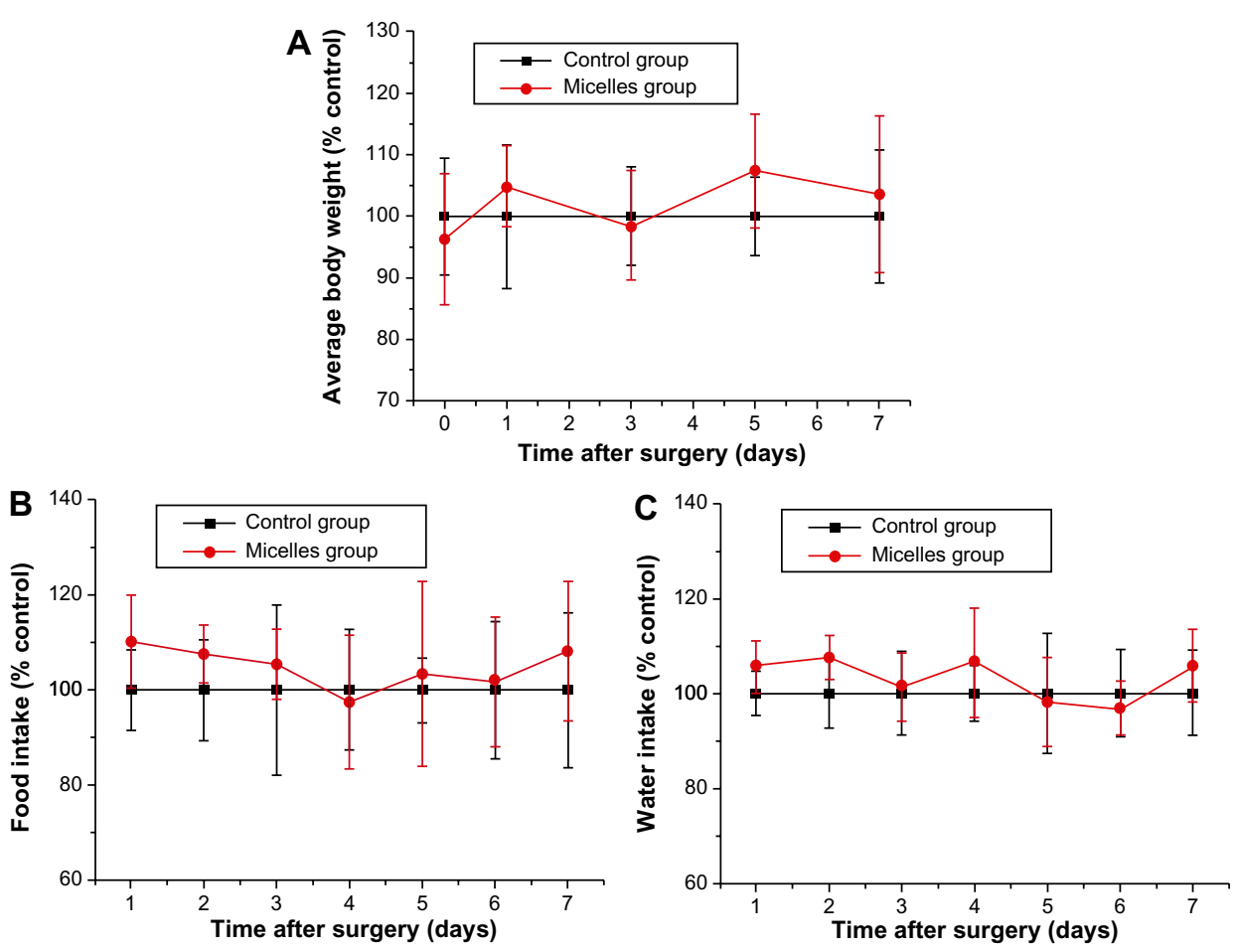

Figure 8 (A) Body weight, (B) food intake, and (C) water intake of mice in each group after surgery.

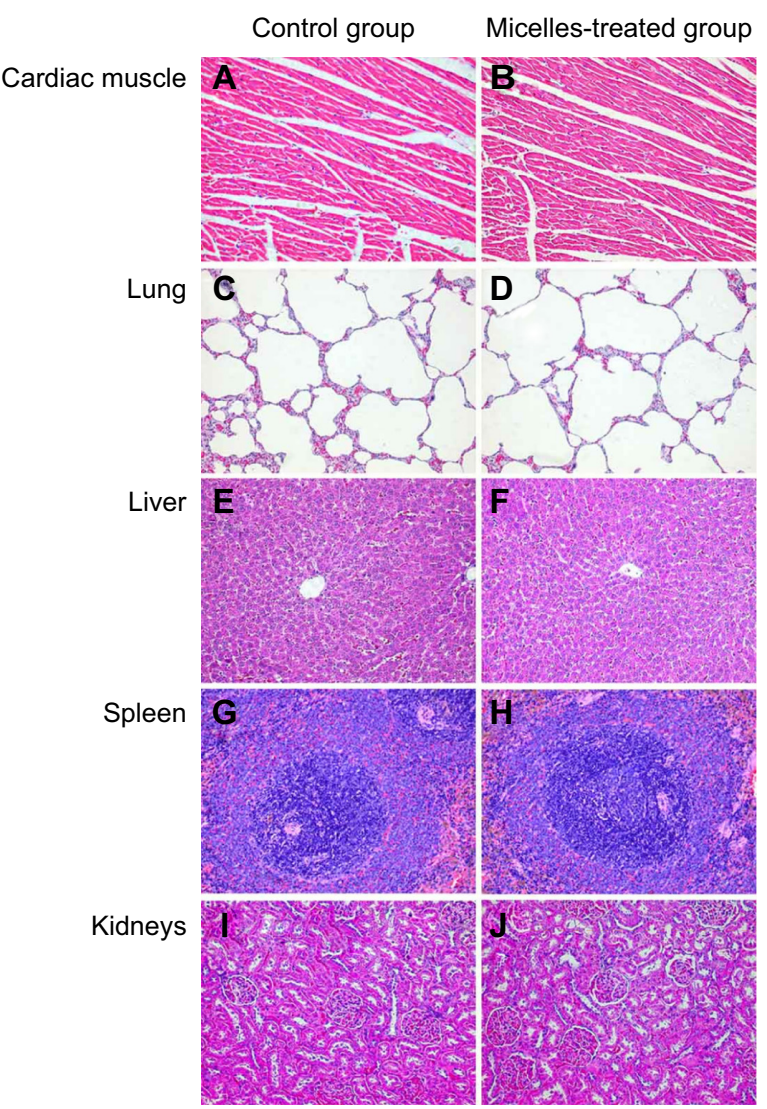

Figure 9 Photographs of mouse major organs after administration of micelles (200x) on Day 7 (A-J).
Furthermore, we analyzed histologic changes of major organs, including the heart, lung, liver, spleen, and kidney (Figure 9) on the 7th day after administration. Compared with the NS group, no obvious hematologic changes were observed in H\&E staining of sections of the major organs under microscope.

\section{Conclusion}

In this work, we prepared and characterized biodegradable and thermosensitive PCL-PEG-PCL micelles. The micelles were free-flowing sol at low temperature, but instantly converted into nonflowing gel at body temperature. The thermosensitive micelles could reduce the severity and even prevent formation of ischemia-induced adhesion. In vitro and in vivo experiments suggested that the thermosensitive micelles are biocompatible with low cytotoxicity. The results indicated that the PCL-PEG-PCL micelles could serve as a potential barrier agent to prevent ischemia-induced adhesion after surgery.

\section{Acknowledgments}

This work was financially supported by National Natural Science Foundation (NSFC81201724), Sichuan Support Project of Science and Technology (2013SZ0018), Specialized Research Fund for the Doctoral Program of Higher 
Education (20120181120044), Distinguished Young Scholars of Sichuan University (2013SCU04A16), and China Postdoctoral Science Foundation (2013M531969).

\section{Disclosure}

The authors report no conflicts of interest in this work. The authors alone are responsible for the content and writing of the paper.

\section{References}

1. Zong X, Li S, Chen E, et al. Prevention of postsurgery-induced abdominal adhesions by electrospun bioabsorbable nanofibrous poly(lactide-coglycolide)-based membranes. Ann Surg. 2004;240(5):910-915.

2. Gong CY, Wu QJ, Liao JF, et al. Prevention of postsurgical cauterizationinduced peritoneal adhesions by biodegradable and thermosensitive micelles. J Biomed Nanotechnol. 2013;9(12):1984-1995.

3. ten Broek RP, Wilbers J, van Goor H. Electrocautery causes more ischemic peritoneal tissue damage than ultrasonic dissection. Surg Endosc. 2011;25(6):1827-1834.

4. Ahn HS, Lee H-J, Yoo M-W, et al. Efficacy of an injectable thermosensitive gel on postoperative adhesion in rat model. $J$ Korean Surg Soc. 2010;79(4):239-245.

5. Liakakos T, Thomakos N, Fine PM, Dervenis C, Young RL. Peritoneal adhesions: etiology, pathophysiology, and clinical significance. Recent advances in prevention and management. Dig Surg. 2001;18(4): 260-273.

6. Diamond MP. Reduction of de novo postsurgical adhesions by intraoperative precoating with Sepracoat (HAL-C) solution: a prospective, randomized, blinded, placebo-controlled multicenter study. The Sepracoat Adhesion Study Group. Fertil Steril. 1998;69(6):1067-1074.

7. Yang B, Gong CY, Zhao X, et al. Preventing postoperative abdominal adhesions in a rat model with PEG-PCL-PEG hydrogel. Int $J$ Nanomedicine. 2012;7:547-557.
8. Liu Y, Li H, Shu XZ, Gray SD, Prestwich GD. Crosslinked hyaluronan hydrogels containing mitomycin $\mathrm{C}$ reduce postoperative abdominal adhesions. Fertil Steril. 2005;83 Suppl 1:1275-1283.

9. Gong C, Wang C, Wang Y, et al. Efficient inhibition of colorectal peritoneal carcinomatosis by drug loaded micelles in thermosensitive hydrogel composites. Nanoscale. 2012;4(10):3095-3104.

10. Gong C, Qi T, Wei X, et al. Thermosensitive polymeric hydrogels as drug delivery systems. Curr Med Chem. 2013;20(1):79-94.

11. Gao X, Deng X, Wei X, et al. Novel thermosensitive hydrogel for preventing formation of abdominal adhesions. Int $J$ Nanomedicine. 2013;8:2453-2463.

12. Gong C, Wu Q, Wang Y, et al. A biodegradable hydrogel system containing curcumin encapsulated in micelles for cutaneous wound healing. Biomaterials. 2013;34(27):6377-6387.

13. Gong C, Yang B, Qian Z, et al. Improving intraperitoneal chemotherapeutic effect and preventing postsurgical adhesions simultaneously with biodegradable micelles. Nanomedicine. 2012;8(6):963-973.

14. Wu Q, Gong C, Shi S, et al. Mannan loaded biodegradable and injectable thermosensitive PCL-PEG-PCL hydrogel for vaccine delivery. Soft Materials. 2012;10(4):472-486.

15. Reed KL, Fruin AB, Gower AC, Stucchi AF, Leeman SE, Becker JM. A neurokinin 1 receptor antagonist decreases postoperative peritoneal adhesion formation and increases peritoneal fibrinolytic activity. Proc Natl Acad Sci U S A. 2004;101(24):9115-9120.

16. Reed KL, Fruin AB, Bishop-Bartolomei KK, et al. Neurokinin-1 receptor and substance $P$ messenger RNA levels increase during intraabdominal adhesion formation. J Surg Res. 2002;108(1):165-172.

17. Nishioka Y, Miyazaki M, Abe K, et al. Regeneration of peritoneal mesothelium in a rat model of peritoneal fibrosis. Ren Fail. 2008;30(1): 97-105.

18. Mutsaers SE. Mesothelial cells: their structure, function and role in serosal repair. Respirology. 2002;7(3):171-191.

19. Mutsaers SE. The mesothelial cell. Int J Biochem Cell B. 2004;36(1): 9-16.
International Journal of Nanomedicine

\section{Publish your work in this journal}

The International Journal of Nanomedicine is an international, peerreviewed journal focusing on the application of nanotechnology in diagnostics, therapeutics, and drug delivery systems throughout the biomedical field. This journal is indexed on PubMed Central, MedLine, CAS, SciSearch $\AA$, Current Contents ${ } /$ Clinical Medicine,

\section{Dovepress}

Journal Citation Reports/Science Edition, EMBase, Scopus and the Elsevier Bibliographic databases. The manuscript management system is completely online and includes a very quick and fair peer-review system, which is all easy to use. Visit http://www.dovepress.com/ testimonials.php to read real quotes from published authors. 